\title{
Endoplasmic reticulum protein 29 is involved in endoplasmic reticulum stress in islet beta cells
}

\author{
JIALIN GAO ${ }^{1,2}$, YAO ZHANG $^{2}$, LIZHUO WANG $^{2}$, LIBING XIA ${ }^{1}$, MEIQING LU ${ }^{1}$, \\ BINGHUA ZHANG $^{1}$, YUEPING CHEN $^{1}$ and LIANZHI HE ${ }^{3}$
}

\begin{abstract}
${ }^{1}$ Department of Endocrinology and Genetic Metabolism, Yijishan Hospital of Wannan Medical College, Wuhu, Anhui 241002;
${ }^{2}$ Anhui Province Key Laboratory of Biological Macromolecular Research, Wannan Medical College, Wuhu, Anhui 241001;

${ }^{3}$ Department of Gynaecology and Obstetrics, Yijishan Hospital of Wannan Medical Collage, Wuhu, Anhui 241002, P.R. China
\end{abstract}

Received January 1, 2015; Accepted September 25, 2015

DOI: $10.3892 / \mathrm{mmr} .2015 .4527$

\begin{abstract}
Endoplasmic reticulum stress (ERS) is correlated with insulin resistance and islet-cell function. In the present study, the sub-cellular localization and role of ER protein 29 (Erp29) were investigated in an in vitro ERS model of islet beta cells. The INS-1 islet cell line was treated with various concentrations of tunicamycin to establish the ERS model. Immunofluorescence microscopy demonstrated that Erp29 and anti-ER marker protein calnexin were co-localized in NIH3T3 cells, suggesting that Erp29 is localized to the ER. The ERS model induced by tunicamycin showed significantly increased expression of binding immunoglobulin protein (BIP)/glucose-regulated protein 78 (Grp78), which is a marker for ERS, and the expression of Erp29 was also increased at the mRNA and protein levels. Of note, ERS was blocked following small interfering RNA-mediated silencing of Erp29 expression, as indicated by reduced BIP/Grp78 expression. As an ER protein, Erp29 may have an important role in ERS in islet beta cells.
\end{abstract}

\section{Introduction}

Insulin resistance and declining function of pancreatic islet beta cells result in diabetes mellitus type 2 . It was first proposed in 2004 that endoplasmic reticulum stress (ERS) is closely associated with insulin resistance and islet-cell

Correspondence to: Professor Jialin Gao, Department of Endocrinology and Genetic Metabolism, Yijishan Hospital of Wannan Medical College, 2 West Zheshan Road, Wuhu, Anhui 241002, P.R. China

E-mail: jialing.gao@yahoo.com

Professor Lianzhi He, Department of Gynaecology and Obstetrics, Yijishan Hospital of Wannan Medical Collage, 2 West Zheshan Road, Wuhu, Anhui 241002, P.R. China

E-mail: 429094335@qq.com

Key words: endoplasmic reticulum protein 29, endoplasmic reticulum stress, islet beta cell dysfunction, and ultimately diabetes $(1,2)$. ERS-induced apoptosis was also shown to contribute to insulin resistance (3), and intervention in ERS is therefore a potential strategy for diabetes treatment. Erp29 is a protein located in the ER membrane of numerous tissue types and is highly evolutionarily conserved in mammals, although its exact functions have remained elusive (4) and it has yet to be clarified whether Erp29 is involved in ERS in islet beta cells. Misfolded proteins trigger a specific stress response, which is referred to as the unfolded protein response (UPR) and has an important role in the development of ERS. One of the main targets of Erp29 is glucose-regulated protein 78 (Grp78) (5,6), which forms a chaperone and signaling regulator complex with binding immunoglobulin protein (BIP) that monitors ERS (7). In the present study, an ERS model of islet beta cells was established and used to explore the role of Erp29 in ERS in islet beta cells.

\section{Materials and methods}

Antibodies (Abs). The following primary antibodies were used in the present study: Anti-rat BIP/Grp78 rabbit monoclonal Ab (cat no. ab32618; Abcam, Cambridge, MA, USA), anti-Erp29 goat polyclonal Ab (cat no. EB08977; Everest Biotech, Heyford, UK), anti-calnexin rabbit polyclonal Ab (ER marker; cat no. ab22595; Abcam), anti-GAPDH mouse polyclonal Ab (cat no. AG019; Beyotime Institute of Biotechnology, Haimen, China), anti- $\beta$-actin mouse monoclonal antibody (cat no. AA128, Beyotime Institute of Biotechnology), Alexa Fluor 488- and 594-conjugated secondary Ab (cat. nos. A11055 and A21442; Invitrogen; Thermo Fisher Scientific, Waltham, MA, USA) and the horseradish peroxidase (HRP)-conjugated secondary Abs goat anti-rabbit (cat no. A0218), goat anti-mouse (cat no. A0216) and donkey anti-goat (cat no. A0181) were all obtained from Beyotime Institute of Biotechnology. All secondary antibodies used for immunofluorescence showed minimal cross-reactivity with other species.

Cell culture. Unless otherwise indicated, all chemicals in the present study were purchased from Sigma-Aldrich (St. Louis, MO, USA). INS-1 cells (provided by the Institute 
of Biochemistry and Cell Biology, Academy of Sciences of China, Shanghai, China) were routinely grown in a humidified atmosphere containing $5 \% \mathrm{CO}_{2}$ and $95 \%$ air at $37^{\circ} \mathrm{C}$ in RPMI-1640 medium (Thermo Fisher Scientific) containing $11.1 \mathrm{mM}$ glucose supplemented with $10 \%$ FBS (Gibco; Thermo Fisher Scientific), $1 \mathrm{mM}$ sodium pyruvate, $10 \mathrm{mM}$ 4-(2-hydroxyethyl)-1-piperazineethanesulfonic acid, $55 \mu \mathrm{M}$ beta-mercaptoethanol, $100 \mathrm{U} / \mathrm{ml}$ penicillin and $100 \mu \mathrm{g} / \mathrm{ml}$ streptomycin. Cells were passaged weekly after detachment with trypsin-EDTA, and all experiments were performed between passages 4 and 20 .

Immunofluorescence microscopy. INS-1 cells were grown on coverslips in 24-well plates, washed twice with phosphate-buffered saline (PBS) and fixed with $4 \%$ paraformaldehyde for 10-30 min. After two more washes with PBS, cells were permeabilized/blocked with PBS containing $0.2 \%$ saponin and $10 \%$ bovine serum albumin (SS-PBS) for $30 \mathrm{~min}$. For double immunostaining, coverslips were incubated with primary Abs followed by secondary Abs in SS-PBS for $1 \mathrm{~h}$ and $30 \mathrm{~min}$ each, followed by three washes with PBS. Coverslips were mounted onto 1-mm glass slides using fluorescent mounting medium. All steps were performed at room temperature and samples were analyzed using a Leica TCS SP2 confocal microscope (Leica Microsystems, Wetzlar, Germany).

Erp29 siRNA and establishment of INS-1-cell model of ERS. The specific siRNA for Erp29 (sense, 5'-ACGCUU AAACUCAUCUUGCUU-3' and antisense, 5'-GCAAGA UGAGUUUAAGCGUCU-3') and non-target control siRNA (sense, 5'-UUCUCCGAACGUGUCACGUTT-3' and antisense, 5'-ACGUGACACGUUCGGAGAATT-3') were used in the current study (Shanghai GenePharma Co., Ltd., Shanghai, China). Cells were transfected with siRNA using Lipofectamine 2000 (Invitrogen; Thermo Fisher Scientific) according to the manufacturer's instructions. To induce ER stress in vitro, INS-1 cells in the logarithmic growth phase were cultured overnight in the presence of $0,2,5$, 10 and $20 \mu \mathrm{g} / \mathrm{ml}$ tunicamycin (Sigma-Aldrich).

Reverse-transcription polymerase chain reaction (RT-PCR) analysis. PCR was performed using a panel of normalized cDNAs prepared from treated INS-1 cells using TRIzol reagent (Invitrogen; Thermo Fisher Scientific). Three different internal fragments were amplified using an Applied Biosystems 9700 PCR machine (Thermo Fisher Scientific) using the following primers: Erp29 sense, 5'-AACCCG ATTCCTGCTTTG-3' and anti-sense, 5'-TTGCCTTGA ACACCACCT-3', (750-bp fragment); GRP78 sense, 5'-TCA GCCCACCGTAACAAT-3' and anti-sense, 5'-CAAACT TCTCGCGTCAT-3' (275-bp fragment). Amplification was performed over 33-35 cycles with an annealing temperature of $56-62^{\circ} \mathrm{C}$. Normalization to GAPDH was performed, which was amplified using the following primers: Sense, 5'-GAA GGTCGGAGTCCACGG-3' and anti-sense, 5'-GAATGG TGATGGGATT-3' (221-bp fragment). All PCR primers were produced by Sangon Biotech, Co., Ltd. (Shanghai, China). A PCR mix kit (Tiangen Biotech Co., Ltd., Beijing, China) was used in the PCR protocol. The $25 \mu 1$ total reaction volume contained $12.5 \mu \mathrm{l} 2 \mathrm{X}$ GC buffer, $1 \mu \mathrm{l}$ dNTPs, $1 \mu \mathrm{l}$ primers, $1 \mu \mathrm{l}$ cDNA, $0.25 \mu \mathrm{l}$ Taq polymerase and $7.75 \mu \mathrm{l}$ $\mathrm{ddH}_{2} \mathrm{O}$. The PCR cycling conditions were as follows: $94^{\circ} \mathrm{C}$ for $4 \mathrm{~min}$, then a total of 32 cycles of $94^{\circ} \mathrm{C}$ for $45 \mathrm{sec}$, $60 / 56 / 58.5^{\circ} \mathrm{C}$ (Grp78/Erp29/GAPDH) for $45 \mathrm{sec}$ and $72^{\circ} \mathrm{C}$ for $60 \mathrm{sec}, 32$ cycles, then $72^{\circ} \mathrm{C}$ for $10 \mathrm{~min}$. The PCR products were separated by agarose gel electrophoresis, and the bands were analyzed by Gray analysis software (Quantity One 4.5; Bio-Rad Laboratories, Inc., Hercules, CA, USA). The DNA ladder was obtained from Beyotime Institute of Biotechnology.

Western blot analysis. Protein was extracted from treated INS-1 cells and the concentrations were determined using the Bio-Rad protein assay (Bio-Rad Laboratories, Inc.). Equal amounts of protein were subjected to $10 \%$ SDS-PAGE and transferred onto polyvinylidene fluoride membranes (EMD Millipore, Billerica, MA, USA). The blots were blocked in $5 \%$ nonfat milk for $1 \mathrm{~h}$ at room temperature. Then the membranes were incubated overnight at $4^{\circ} \mathrm{C}$ with primary antibodies (Erp29 and Grp78, 1:1,000; GAPDH and actin 1:2,000) and then washed three times (10 min each) with Tris-buffered saline (Sigma-Aldrich) containing $0.1 \%$ Tween 20 (Sigma-Aldrich). Subsequently, membranes were incubated with HRP-conjugated secondary antibodies $(1: 5,000)$ for $1 \mathrm{~h}$ at room temperature and immunoreactive proteins were detected by enhanced chemiluminescence reagent (Beyotime Institute of Biotechnology). The blots were then exposed to an X-OMAT AR X-ray film (Kodak, Rochester, NY, USA) for between 10 sex and $5 \mathrm{~min}$ as previously described (8).

Statistical analysis. Values are expressed as the mean \pm standard error of the mean and statistically significant differences between two groups were analyzed using the unpaired Student's t-test. SPSS software, version 13.0 (SPSS, Inc., Chicago, IL, USA) was used for statistical analysis. $\mathrm{P}<0.05$ was considered to indicate a statistically significant difference between values.

\section{Results}

Erp29 is localized to the ER. Immunofluorescence microscopy was used to confirm that Erp29 is localized to the ER using an ER marker protein-specific antibody. Staining for Erp29 (green fluorescence) was observed throughout the cytoplasm, but predominantly in the perinuclear region, whereas no staining was present at the plasma membrane or in the nucleus (Fig. 1A and D). This suggested that Erp29 protein is localized to an intracellular compartment. Co-staining for ER marker protein calnexin (red fluorescence; Fig. 1B and E) indicated co-localization with Erp29 (Fig. 1C and F), further indicating that Erp29 is localized to the ER.

Tunicamycin-induced ERS increases the expression of BIP/Grp78 and Erp29 in INS-1 cells. Ins-1 cells in the logarithmic growth phase (Fig. 2A) were incubated with various concentrations of tunicamycin to induce ERS, and Bip/GRp78 mRNA levels were assessed by RT-PCR analysis (Fig. 2). BIP/Grp78 expression was found to be upregulated 

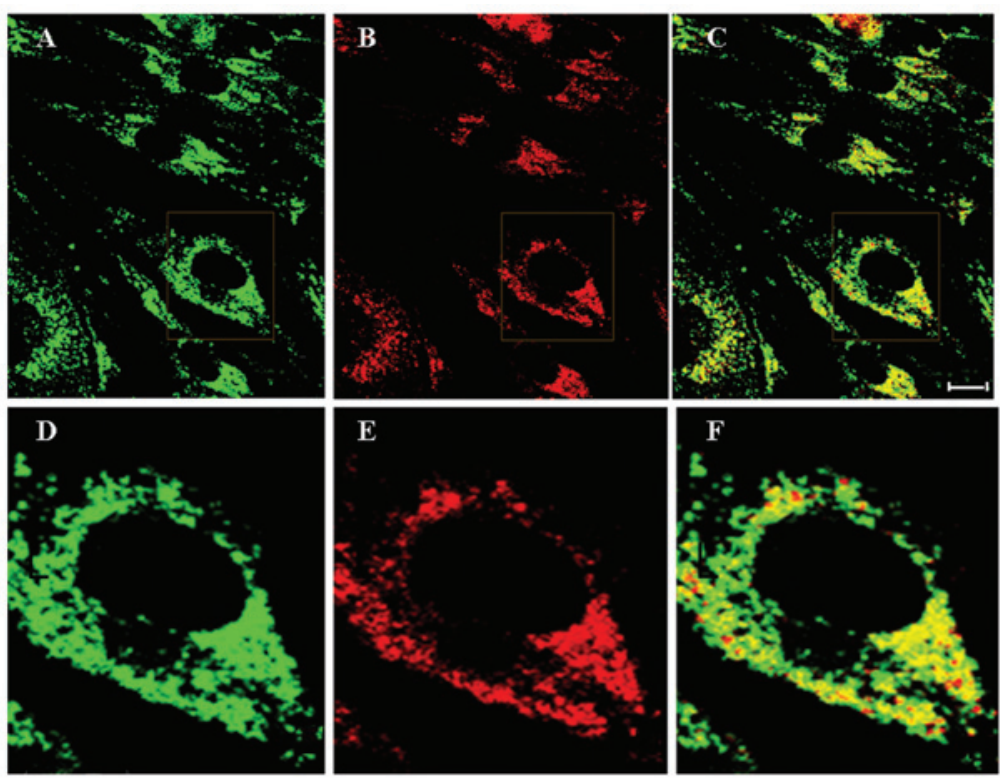

Figure 1. Endoplasmic reticulum localization of Erp29. Erp29 co-localizes with endoplasmic reticulum marker protein calnexin. NIH3T3 cells were fixed, permeabilized and incubated with antibodies against (A) Erp29 (green fluorescence) and (B) calnexin (red fluorescence). Primary antibodies were detected with Alexa flour 488- and 594-labeled secondary antibodies. (C) Overlay of A and B. Scale bar $=10 \mu \mathrm{m}$. Magnification, x400. (D-F) Magnified windows from A, B and C, respectively. Erp, endoplasmic reticulum protein.

with increasing tunicamycin concentration. A tunicamycin concentration of $5 \mu \mathrm{g} / \mathrm{ml}$ and above significantly increased the mRNA expression of BIP/Grp78 $(\mathrm{P}<0.05$ or $\mathrm{P}<0.01$; Fig. 2B and C). Furthermore, Erp29 mRNA expression was enhanced with increasing tunicamycin concentration. Tunamycin at $2 \mu \mathrm{g} / \mathrm{ml}$ and above significantly increased the expression of Erp29 compared with that in the control group $(\mathrm{P}<0.05$ or $\mathrm{P}<0.01$; Fig. $2 \mathrm{~B}$ and $\mathrm{C})$. Furthermore, the protein levels of BIP/Grp78 and Erp29 were assessed by western blot analysis (Fig. 3). In accordance with the changes in mRNA expression, a tunicamycin dose of $2 \mathrm{mg} / \mathrm{ml}$ and above significantly increased the protein expression of BIP/Grp78 compared with that in the control group $(\mathrm{P}<0.01)$ (Fig. 3). As BIP/Grp78 is a marker for ERS its upregulation indicated that ERS had been induced. Furthermore, the protein levels of Erp29 were also significantly increased compared with those in the control group following tunicamycin treatment at $5 \mu \mathrm{g} / \mathrm{ml}$ and above $(\mathrm{P}<0.01)$ (Fig. 3). Upregulation of Erp29 may be associated with ERS in beta cells.

Knockdown of Erp29 decreases BIP/Grp78 expression in tunicamycin-induced ins-1 cells. In order to further investigate the association between Erp29 and ERS, Erp29 expression was silenced using a small interfering (si)RNA approach. After it had been confirmed that Erp29 was successfully silenced (Fig. 4A), it was further identified that ERS responses were reduced in tunicamycin-induced INS-1 cells transfected with Erp29 siRNA, as indicated by a decrease in the protein levels of BIP/Grp78 (Fig. 4B).

\section{Discussion}

Erp29 is an ER protein that is widely expressed in mammalian tissues; however, its exact functions have remained elusive (4). The highly evolutionarily conserved human Erp29
$\mathbf{A}$

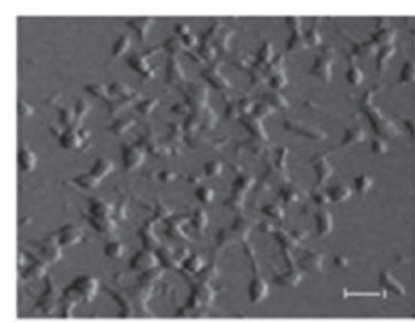

B

Ladder $\begin{array}{lllll}1 & 2 & 3 & 4 & 5\end{array}$

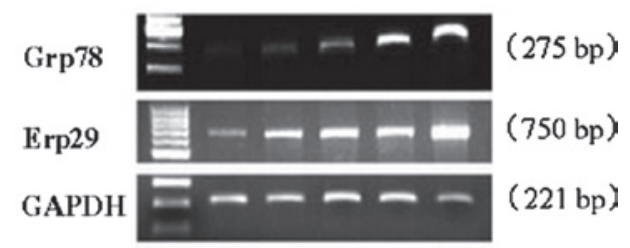

C

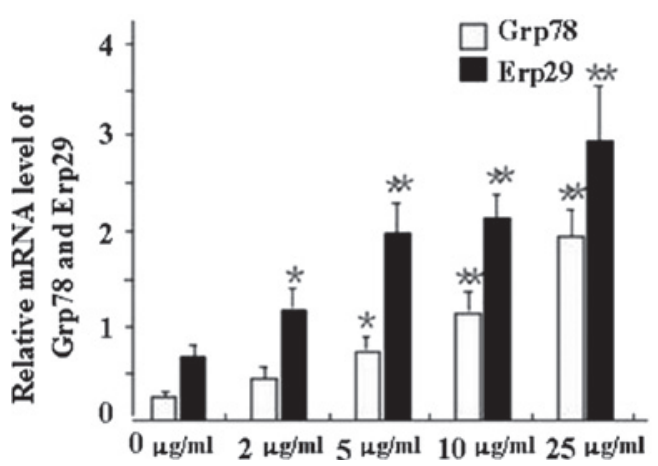

Figure 2. ERS affects mRNA levels of BIP/Grp78 and Erp29 in INS-1 cells. (A) Cultured INS-1 cells (magnification, $\mathrm{x} 100$. Scale bar $=15 \mu \mathrm{m}$ ). (B) Increased mRNA expression of Grp78 (275 bp) and Erp29 (750 bp) in an ERS model of INS-1 cells. Lanes 1-5 show samples treated with 0, 2, 5, 10 and $20 \mu \mathrm{g} / \mathrm{ml}$ tunicamycin, respectively. (C) Quantified results from B normalized to GAPDH. Values are expressed as the mean \pm standard deviation. ${ }^{*} \mathrm{P}<0.05$; ${ }^{* *} \mathrm{P}<0.01$ vs. $0 \mu \mathrm{g} / \mathrm{ml}$. Erp, endoplasmic reticulum protein; Grp, glucose-regulated protein; BIP, binding immunoglobulin protein; ERS, endoplasmic reticulum stress. 
A

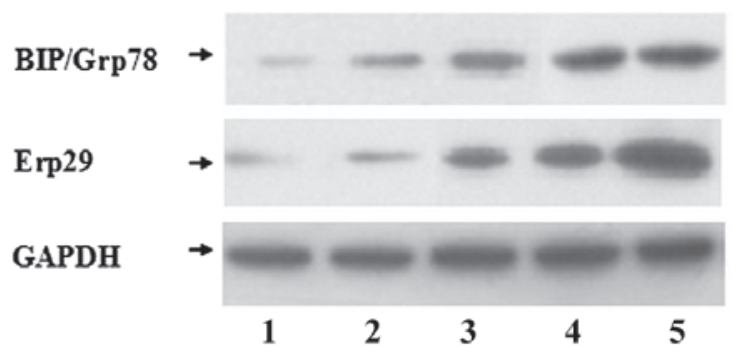

B

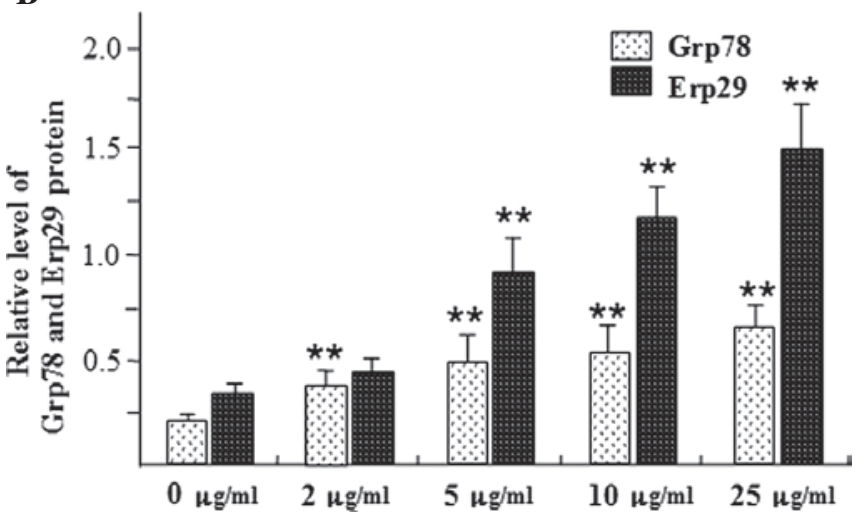

Figure 3. Protein levels of BIP/Grp78 and Erp29 in a INS-1-cell model of endoplasmic reticulum stress. (A) Tunicamycin increased the expression of BIP/Grp78 and Erp29 in INS-1 cells. Lanes 1-5 show samples treated with $0,2,5,10$ and $20 \mu \mathrm{g} / \mathrm{ml}$ tunicamycin, respectively. (B) Quantified results from A normalized to GAPDH. Values are expressed as the mean \pm standard deviation. ${ }^{* *} \mathrm{P}<0.01$ vs. $0 \mu \mathrm{g} / \mathrm{ml}$. Erp, endoplasmic reticulum protein; Grp, glucose-regulated protein; BIP, binding immunoglobulin protein.

gene is located on chromosome 12 and the encoded protein $(29 \mathrm{kDa})$ consists of 261 amino acid residues (4). It has been suggested that Erp29 may be involved in protein folding and transport under ERS conditions (9). ERS-mediated apoptosis in islet cells has an important role in the development of diabetes (10). Pancreatic $\beta$ cells specifically regulate insulin-secreting cells and the highly developed ER in these cells is particularly sensitive to ERS. Following stimulation of insulin secretion by elevation of blood glucose, the released insulin activates further proinsulin biosynthesis in the ER of beta cells. Specifically, preproinsulin is synthesized and the 16-amino acid signaling peptide is recognized by the signal recognition particle, which triggers synergistic translocation into the ER lumen. Following cleavage of the signal peptide to produce proinsulin, the peptide is folded in the ER lumen, transported to the Golgi apparatus and packaged into secretory granules. Finally, proinsulin is converted to the mature form and eventually released by exocytosis. The ER of beta cells is highly sensitive to fluctuations in blood glucose and this signaling affects the folding of proinsulin. Upon entering the ER lumen, insulin affects the ER protein folding capacity as well as $\mathrm{Ca}^{2+}$ homeostasis, which leads to beta cell damage, apoptosis and ultimately ERS (11).

In the present study, double-staining immunofluorescence microscopy was used to confirm the ER localization of Erp29. In addition, a tunicamycin-induced in vitro cell model of ERS was established in islet beta cells. Erp29 as well as the ER chaperone and marker BIP/Grp78 were increased in tunicamycin-induced beta cells, confirming that the model of ERS had been successfully established. Erp29 expression
$\mathbf{A}$

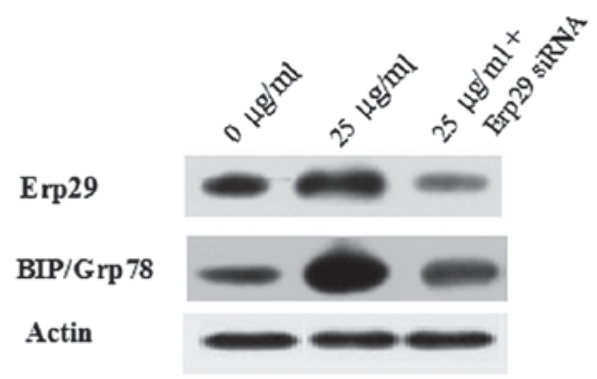

B

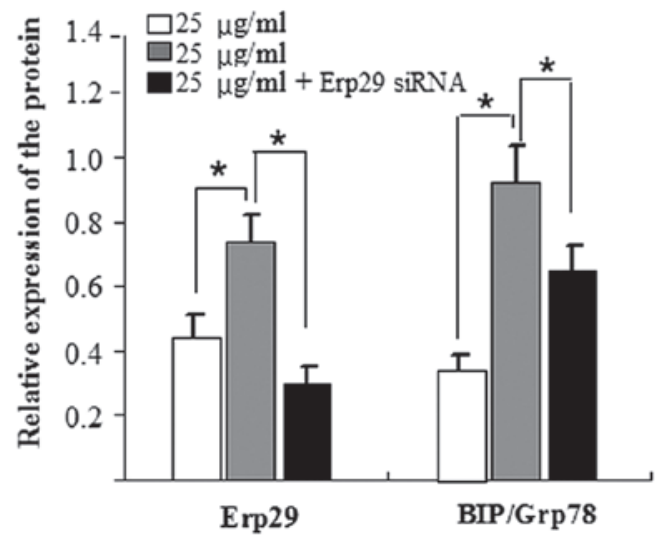

Figure 4. BIP/Grp78 expression under endoplasmic reticulum stress is downregulated following silencing of Erp29. (A) ERP29 expression in INS-1 cells treated with tunicamycin was significantly decreased by Erp29 siRNA. In parallel, BIP/Grp78 was also downregulated by Erp29 siRNA. (B) Quantified results from A normalized to actin. Values are expressed as the mean \pm standard deviation. ${ }^{*} \mathrm{P}<0.05$ vs. $0 \mu \mathrm{g} / \mathrm{ml}$ tunicamycin group. Erp, endoplasmic reticulum protein; Grp, glucose-regulated protein; BIP, binding immunoglobulin protein; siRNA, small interfering RNA.

increased with increasing tunicamycin dose, suggesting that Erp29 is associated with ERS. While its exact role remains elusive and requires further study, Erp29 may serve as a protective factor against net stress in the ER and may act synergistically with BIP/Grp78 to facilitate protein transport from the ER. This would alleviate ERS and promote islet-cell survival, thus avoiding the onset of apoptosis. This result is similar to the findings of a study which assessed Erp29 expression in an IEC26-cell model of radiation injury (12). The present study therefore concluded that Erp29 may be involved in ERS in islet cells and possibly other cell types and tissues. The results indicated that a correlation between Erp29 and cell injury may exist, which requires further study.

\section{Acknowledgements}

The present study was supported by grants from the National Natural Science Foundation of China (nos. 81200632 and 81471002), the Natural Science Foundation of Anhui, China (no. 1308085QH134) and the Introduction of Talents Foundation of Yijishan Hospital (no. YR201104).

\section{References}

1. Ozcan U, Cao Q, Yilmaz E, Lee AH, Iwakoshi NN, Ozdelen E, Tuncman G, Görgün C, Glimcher LH and Hotamisligil GS: Endoplasmic reticulum stress links obesity, insulin action, and type 2 diabetes. Science 306: 457-461, 2004. 
2. Zhao L, Guo H, Chen H, Petersen RB, Zheng L, Peng A and Huang K: Effect of liraglutide on endoplasmic reticulum stress in diabetes. Biochem Biophys Res Commun 441: 133-138, 2013.

3. Ou HY, Wu HT, Hung HC, Yang YC, Wu JS and Chang CJ: Endoplasmic reticulum stress induces the expression of fetuin-A to develop insulin resistance. Endocrinology 153: 2974-2984, 2012.

4. Sargsyan E, Baryshev M, Backlund M, Sharipo A and Mkrtchian S: Genomic organization and promoter characterization of the gene encoding a putative endoplasmic reticulum chaperone, ERp29. Gene 285: 127-139, 2002.

5. Barnes JA and Smoak IW: Glucose-regulated protein 78 (GRP78) is elevated in embryonic mouse heart and induced following hypoglycemic stress. Anat Embryol (Berl) 202: 67-74, 2000.

6. Qian Y, Falahatpisheh MH, Zheng Y, Ramos KS and Tiffany-Castiglioni E: Induction of $78 \mathrm{kD}$ glucose-regulated protein (GRP78) expression and redox-regulated transcription factor activity by lead and mercury in C6 rat glioma cells. Neurotox Res 3: 581-589, 2001.

7. Ma KX, Chen GW, Shi CY, Cheng FF, Dou H, Feng CC and Liu DZ: Molecular characterization of the glucose-regulated protein 78 (GRP78) gene in planarian Dugesia japonica. Comp Biochem Physiol B Biochem Mol Biol 171: 12-17, 2014.
8. Gao J, Xia L, Lu M, Zhang B, Chen Y, Xu R and Wang L: TM7SF1 (GPR137B): A novel lysosome integral membrane protein. Mol Biol Rep 39: 8883-8889, 2012.

9. Sargsyan E, Baryshev M, Szekely L, Sharipo A and Mkrtchian S Identification of ERp29, an endoplasmic reticulum lumenal protein, as a new member of the thyroglobulin folding complex. J Biol Chem 277: 17009-17015, 2002.

10. Marchetti P, Bugliani M, Lupi R, Marselli L, Masini M, Boggi U, Filipponi F, Weir GC, Eizirik DL and Cnop M: The endoplasmic reticulum in pancreatic beta cells of type 2 diabetes patients. Diabetologia 50: 2486-2494, 2007.

11. Zhu M, Guo M, Fei L, Pan XQ and Liu QQ: 4-phenylbutyric acid attenuates endoplasmic reticulum stress-mediated pancreatic beta-cell apoptosis in rats with streptozotocin-induced diabetes. Endocrine 47: 129-137, 2014.

12. Zhang B, Wang M, Yang Y, Wang Y, Pang X, Su Y, Wang J, Ai G and Zou Z: ERp29 is a radiation-responsive gene in IEC-6 cell. J Radiat Res 49: 587-596, 2008. 